\title{
Ecosystem service tradeoff analysis reveals the value of marine spatial planning for multiple ocean uses
}

\author{
Crow White ${ }^{a, 1}$, Benjamin S. Halpern ${ }^{b}$, and Carrie V. Kappel ${ }^{b}$ \\ ${ }^{a}$ Bren School of Environmental Science and Management, University of California, Santa Barbara, CA 93106; and ${ }^{b}$ National Center for Ecological Analysis and \\ Synthesis, University of California, Santa Barbara, CA 93101
}

Edited by Peter M. Kareiva, The Nature Conservancy, Seattle, WA, and approved February 2, 2012 (received for review September 1, 2011 )

\begin{abstract}
Marine spatial planning (MSP) is an emerging responsibility of resource managers around the United States and elsewhere. A key proposed advantage of MSP is that it makes tradeoffs in resource use and sector (stakeholder group) values explicit, but doing so requires tools to assess tradeoffs. We extended tradeoff analyses from economics to simultaneously assess multiple ecosystem services and the values they provide to sectors using a robust, quantitative, and transparent framework. We used the framework to assess potential conflicts among offshore wind energy, commercial fishing, and whalewatching sectors in Massachusetts and identify and quantify the value from choosing optimal wind farm designs that minimize conflicts among these sectors. Most notably, we show that using MSP over conventional planning could prevent $>\$ 1$ million dollars in losses to the incumbent fishery and whale-watching sectors and could generate $>\$ 10$ billion in extra value to the energy sector. The value of MSP increased with the greater the number of sectors considered and the larger the area under management. Importantly, the framework can be applied even when sectors are not measured in dollars (e.g., conservation). Making tradeoffs explicit improves transparency in decision-making, helps avoid unnecessary conflicts attributable to perceived but weak tradeoffs, and focuses debate on finding the most efficient solutions to mitigate real tradeoffs and maximize sector values. Our analysis demonstrates the utility, feasibility, and value of MSP and provides timely support for the management transitions needed for society to address the challenges of an increasingly crowded ocean environment.
\end{abstract}

ecosystem-based management | efficiency frontier | multisector planning | bioeconomic model | renewable energy

Coastal waters around the world are experiencing increasing demand for their diverse human benefits, or ecosystem services. Demand comes from existing sectors, such as fishing and transportation, that seek to expand their activities and emerging sectors, such as renewable energy and offshore aquaculture. The need to coordinate these human uses to reduce impacts across sectors is prompting calls for ecosystem-based coastal and marine spatial planning (MSP) (1). In the United States, Executive Order 13547 mandates this approach to marine resource management, and many US states and other countries have recently passed legislation emphasizing MSP (e.g., ref. 2).

Despite mounting interest in MSP, it has been difficult to implement for at least two reasons (3). First, user groups are wary of negative effects of regulatory changes to the status quo, and they legitimately ask for evidence that MSP will generate improvements. Evidence of benefits could include increased management efficiency, greater stakeholder involvement, and outcomes that better achieve management goals. Here, we illustrate how singleand multisector management decisions affect sector values and how MSP (i.e., coordinated multisectoral planning for reducing sector conflicts and increasing their values) can explicitly improve sector values while achieving management goals, thus enhancing potential for stakeholder buy-in.

A second barrier to MSP is that the science for assessing and communicating tradeoffs among human uses of the ocean, and identifying strategies to mediate these tradeoffs, has been slow to catch up with policy opportunities emerging from efforts to implement ecosystem-based management, MSP, and marine pro- tected areas (4). All of these management approaches are fundamentally about making decisions that affect tradeoffs among multiple sectors. However, tradeoffs are rarely addressed explicitly or transparently, and so they often go unrealized or are poorly evaluated. An important proposed advantage of MSP is that it makes tradeoffs explicit, but to do this requires analytical tools for assessing spatial conflicts and synergies among sectors. Economics has a rich history of quantifying and balancing tradeoffs, and resource economics has done so with ecosystem services for over a decade (e.g., refs. 5 and 6), yet this work has not been fully recognized or used explicitly to inform MSP. Here, we draw on this legacy and extend it to provide a robust, quantitative and understandable framework for simultaneously assessing multiple marine ecosystem services and their value to sectors.

Renewable Energy as a Catalyst for MSP. As one of the fastest growing new uses of the ocean, renewable-energy development is catalyzing debate around how we allocate ocean space $(2,7)$. This is particularly true in Massachusetts, which passed the first US law requiring MSP in 2008. Offshore wind-energy development helped motivate creation of this law: wind farms have been proposed for areas where sector crowding is already high, such as the contentious Cape Wind project in Nantucket Sound (7). Recognizing the Massachusetts wind farm debate as an opportunity to demonstrate the feasibility and utility of MSP, we used this example to show the value-added from doing MSP over conventional single-sector management, which focuses on maximizing sectoral values. In particular, we $(i)$ generated alternative wind farm development scenarios driven by single- versus multisector management decisions; (ii) calculated the resulting value of energy and other sectors with which there are spatial conflicts in the marine ecosystem of Massachusetts; (iii) compared sector values arising from alternative development scenarios to show how tradeoffs among sectors can be quantified, and then reduced, by choosing specific MSP scenarios; and (iv) quantified the potential value added to sectors by using MSP over a single-sector approach.

We focused on two provisional wind energy zones (P1 and P2) identified in the Massachusetts Ocean Management Plan to provide proactive results for guiding potential future management decisions (Fig. $1 A$ and $B$ ) (8). We evaluated potential impacts of wind farm installations on two high-value, high-profile sectors: commercial fisheries and whale-watching tourism and conservation. We focused on two iconic and high-value fisheries with different characteristics: the American lobster fishery, which uses fixed gear (traps) on hard-bottom habitat; and the winter flounder fishery, which uses mobile gear (trawls, gillnets) over mostly soft-bottom habitat (Fig. $1 C$ and $D$ ). We bundled whale watching and conservation into a single sector representing the value to society of whales in the wild (Fig. $1 E$ ).

Author contributions: C.W., B.S.H., and C.V.K. designed research, performed research, contributed new reagents/analytic tools, analyzed data, and wrote the paper.

The authors declare no conflict of interest.

This article is a PNAS Direct Submission.

Freely available online through the PNAS open access option.

${ }^{1}$ To whom correspondence should be addressed. E-mail: cwhite@bren.ucsb.edu.

This article contains supporting information online at www.pnas.org/lookup/suppl/doi:10 1073/pnas.1114215109/-/DCSupplemental. 
A

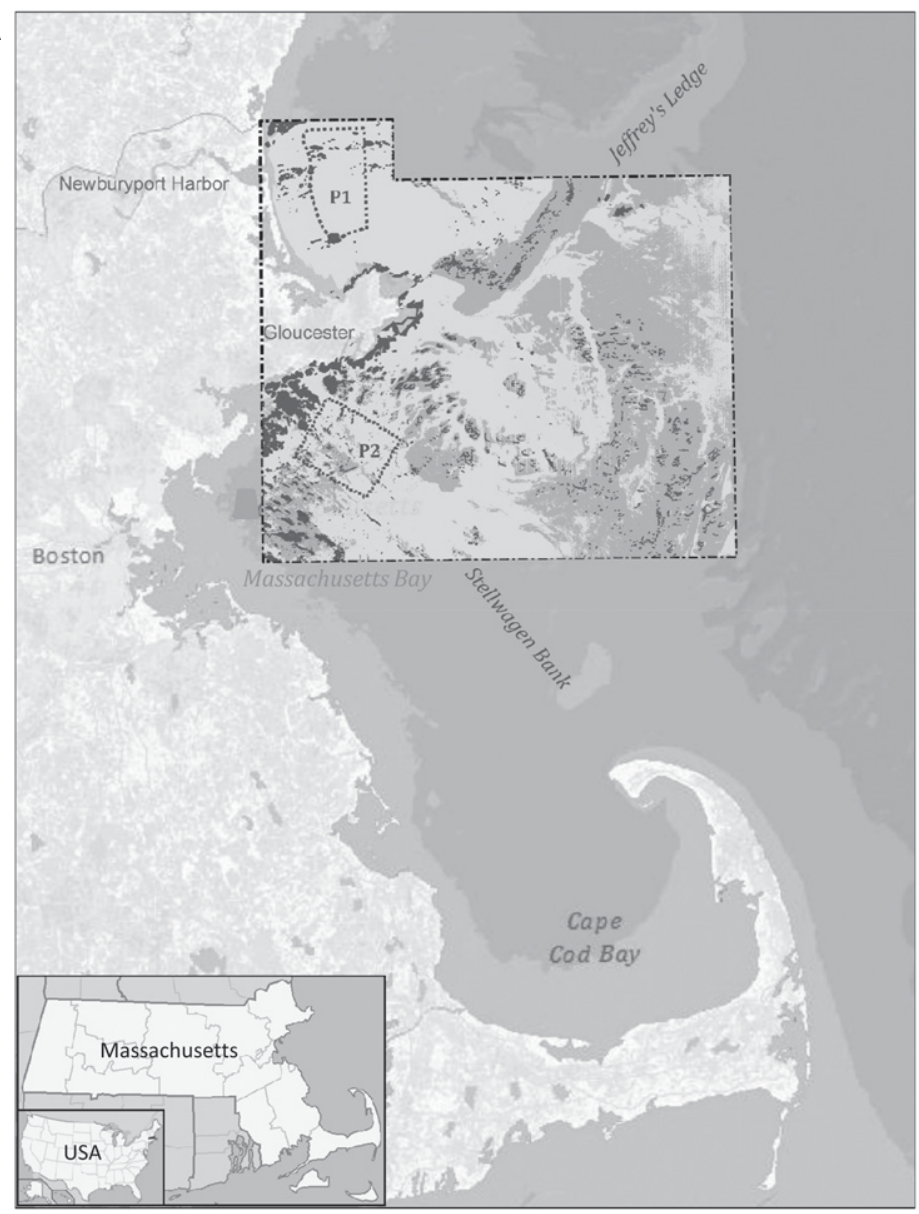

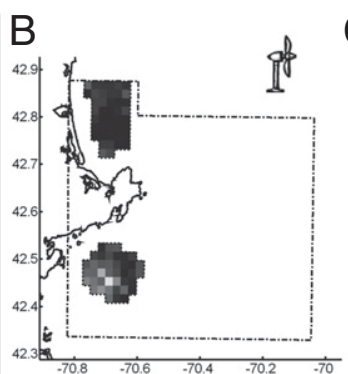

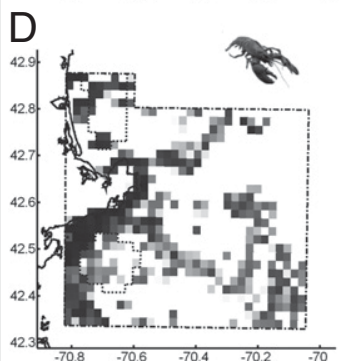

C

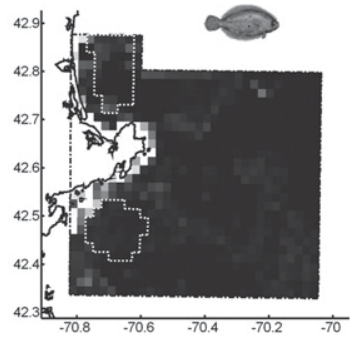

$\mathrm{E}$

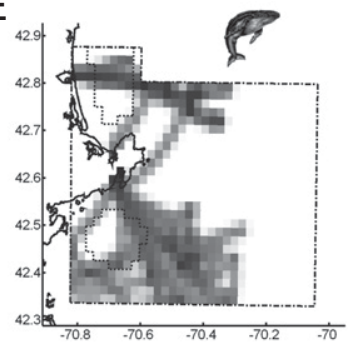

Value (\%)

0 100

\section{Legend for map A}

$\begin{array}{cl}-\cdots & \text { Study area } \\ ------ & \text { Provisional wind energy areas } \\ \square \quad \text { Sand, silt or mud } \\ \square \quad \text { Gravel } \\ \square \quad \text { Rock }\end{array}$

Fig. 1. Massachusetts Bay and spatial distributions of resources and sector values. (A) Habitat distributions. (B-E) Net present values of offshore wind energy, flounder and lobster fishery, and whale-watching sectors, respectively. The value in each grid cell is scaled relative to the maximum absolute value of the sector (based on logged, scaled boat density for the whale sector and profit for the other sectors; see Methods) across all grid cells, in the absence of other sectors.

Each energy zone could feasibly contain hundreds of turbines with the potential to alter fish ecology and constrain fishing patterns, as well as displace endangered whale species and disrupt whale-watching tourism. These incumbent sectors also interact directly and indirectly with each other through the ecosystem, creating potential unanticipated consequences of management action. We explicitly accounted for these intersectoral interactions when we quantified tradeoffs among the sectors under alternative management scenarios, differing in the level of wind energy development and spatial configuration of turbines. We further identified optimal wind farm designs that minimize spatial conflicts and maximize the value of each sector and the joint value of the ecosystem. Finally, and critically, we quantified the sector gains achieved from choosing these optimal solutions, demonstrating the value of MSP.

\section{Results}

Sector Tradeoffs. In simple terms, MSP distributes sectors among their highest-value locations with the lowest intersectoral conflicts $(4,9)$. Here, this means seeking wind-energy areas with both high wind and low fisheries and whale-watching values. Although MSP ultimately requires simultaneous analysis of all sectors, we begin with pairwise tradeoffs between sectors and then progress to three- and four-way analyses.

Borrowing from economics, we visualize tradeoffs by plotting sector values against each other in relation to potential management strategies. These plots reveal the nature and severity of tradeoffs among sectors, enable a given management decision (a point) to be compared with alternative decisions (other points), and allow for easy visualization and measurement of the potential gains from optimal multisector spatial planning (Fig. $2 A$ ). Outcomes from single-sector management serve as a reference against which to measure these gains. Points along the outer boundary of outcomes (the efficiency frontier) represent the set of multisector (ecosystem-based) management strategies that maximize combinations of sector values. Strategies interior to the efficiency frontier can be improved at no cost to either sector, and potential benefit to both, by choosing solutions represented by points closer to or along the frontier.

We used heuristic algorithms to identify optimal strategies delineating the efficiency frontiers (SI Appendix). Although only a few strategies (Fig. $2 E-G$ ) are indicated on each efficiency frontier, a strategy exists for virtually every position along each frontier; these could be found through further computational searching. Sensitivity analysis showed our results to be robust to uncertainty in model parameters characterizing stock-recruitment functions and virgin biomass levels for the fishery species ( $S I$ Appendix, Fig. S4).

Tradeoffs between sectors are clear from our model results (Fig. $2 B-D$ ). Negative-sloped lines indicate significant tradeoffs, and convex frontiers indicate that tradeoffs are not one-to-one. The tradeoff is most severe for the flounder fishery, which directly competes with the energy sector for soft-bottom habitat, and whose mobile gear is permanently excluded from near turbines. Consequently, development of the energy sector to full capacity reduces the value of the flounder fishery within P1 and P2 to zero. Spillover of flounder attributable to a "de facto" reserve effect of the turbines is too small to offset losses. Loss in 
A

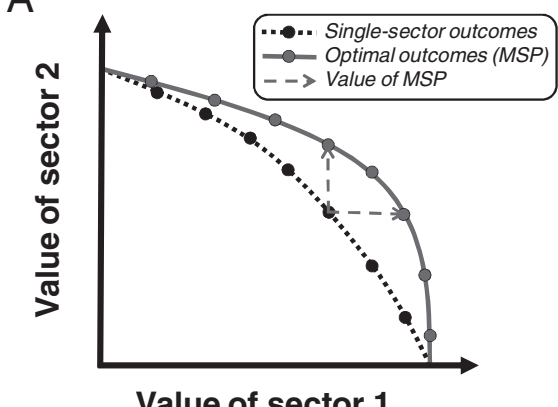

C

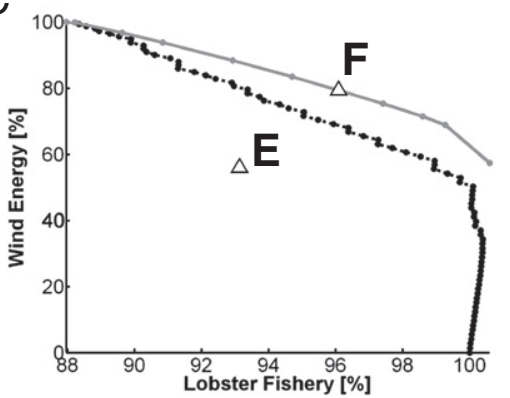

$B$

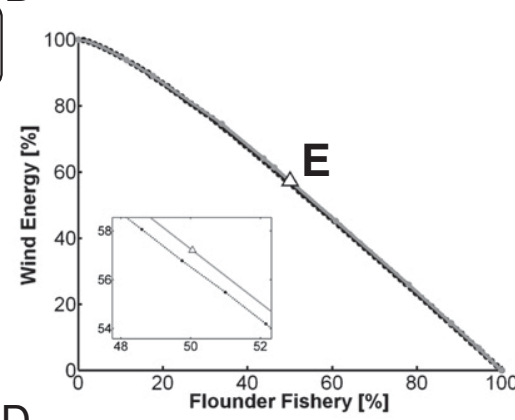

D

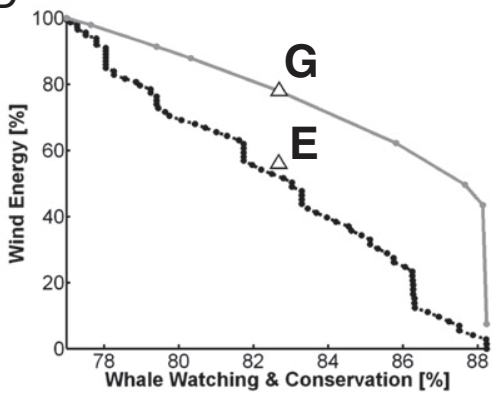

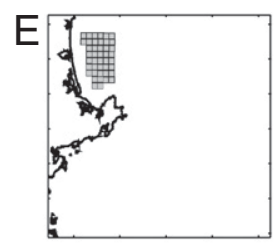

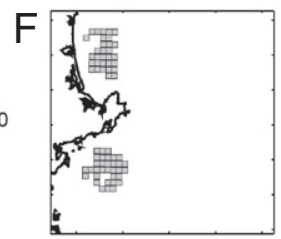

G

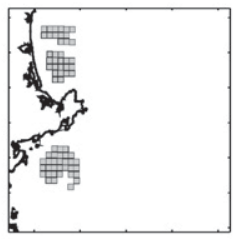

Fig. 2. Pairwise tradeoffs in sector values in relation to spatial management strategies and associated wind farm maps. $(A)$ Conceptual example of sector tradeoffs. Orthogonal dashed lines with arrows illustrate how to measure the value of MSP over single sector management. $(B-D)$ Offshore wind energy flounder and lobster fishery, and whale-watching sector values in relation to wind farm designs in Massachusetts Bay. Sector values are scaled to $100 \%$ at maximum value without any intersectoral conflicts. Lettered triangles correspond with maps of wind energy farms in $E-G$. The inset in $B$ shows a zoomed view for clarity.

percentage value to the lobster fishery is less severe because of less-stringent exclusion regulations around the turbines for this fishery, little natural rocky habitat in the energy zones, and generation of a little additional hard substrate around the turbine foundations. Loss in percentage value to the whale-watching sector is similarly less severe: in this case, boats and whales are only displaced during turbine construction. Note, the whalewatching sector is inherently limited to $\sim 88 \%$ of its maximum value without any intersectoral conflicts because of effects of the existing lobster fishery on whale entanglement and prey availability (herring, used as lobster bait); this tradeoff could be explored explicitly in relation to regulation of lobster fishing to protect whales, but that is beyond the scope of this analysis.

The tradeoff plots also allow us to quantify and compare outcomes of specific proposed wind farm configurations, such as scenario E, which represents complete and exclusive wind farm development within P1. In relation to the flounder fishery and energy sectors (Fig. 2B), E is along the efficiency frontier and thus effective at reducing intersectoral conflicts, to the extent possible. However, in relation to lobster and whale-watching sectors, E lies well below the efficiency frontiers, indicating its inferiority in reducing conflicts compared with what could be achieved using MSP (Fig. $2 C$ and $D$ ).

One can see how MSP produces configurations that reduce spatial conflicts by comparing mapped solutions $E, F$, and $G$ in Fig. 2 with maps of sector values in Fig. $1 B-E$. Solution $E$ is efficient in relation to energy and flounder sectors because patches in the northern zone (P1) are among the highest available in energy value (see also SI Appendix, Fig. S3), whereas patches in the southern zone (P2) are typically more valuable to the flounder fishery. Solution F efficiently mediates the energylobster tradeoff because energy development avoids high-value lobster patches, which are typically closer to shore. Solution G similarly mediates energy-whale sector conflicts; $G$ results in a corridor of undeveloped patches in P1 that allows unobstructed passage by boats to whale-watching sites within the energy zone and at Jeffrey's Ledge. These examples underscore the intent of MSP to rationally allocate multiple ocean uses in a spatially finite environment. However, finding the most efficient solutions for mediating conflicts between even just two sectors is not trivial without analytical support; this support is even more critical for identifying efficient solutions in relation to all sectors in the ecosystem, as we show below.
Optimal Solutions. Truly optimal MSP requires simultaneous consideration of all sectors in the ecosystem. We did this in two stages (Fig. 3). First, we considered a three-way tradeoff in value among energy, whale, and lobster sectors to produce a 3D efficiency frontier surface (Fig. $3 A$ ). Along its edges, the efficiency frontier contains the strategies from the pairwise efficiency frontiers in Fig. $2 C$ and $D$; across the rest of the surface are additional strategies (squares) that maximize values across the three sectors. Selecting a particular management option from the efficiency frontier surface is a political decision, which would be based on the relative preferences of society for maximizing the values of the three sectors.

Second, we extended the tradeoff analysis to consider all four sectors in the ecosystem. Although visualizing the 4D tradeoff is challenging, the analytical process is the same. The four-sector efficiency frontier includes both the three-sector energy-whalelobster efficiency frontier (surface and associated points in Fig. $3 A$ ) and additional strategies that represent optimal combinations in value for all four sectors. The additional strategies do not lie on the three-sector efficiency frontier, and compared with strategies on that frontier, they increase the value of the flounder fishery (because it is now accounted for; Fig. 3B; see SI Appendix, Fig. S5 for full 4D plot). An objective debate around optimal wind farm design in relation to all four sectors should focus on solutions along this comprehensive efficiency frontier.

Value of MSP. Here, we compare the gains to sectors from MSP to outcomes under strategic single-sector management. Single-sector management decisions are initially regulated by the total area within the energy zones that can be developed: the energy sector develops the highest-value patches up to this limit. In response to a particular wind farm design, fishery sectors then strategically adjust fleet effort levels to maximize their values. The whalewatching sector loses value in patches with turbines that cannot be recouped elsewhere. Although we refer to this management scenario as "single-sector," in reality, it has already incorporated some multisector planning: the provisional energy zones were chosen by Massachusetts because they are good wind sites and have fewer potential use conflicts with existing sectors than other possible locations (8). To the degree that this quasi-MSP approach is effective, it provides an improvement over true single-sector management (i.e., no prescreening of development sites). Thus, our assessment of the value of MSP is both realistic for what is 

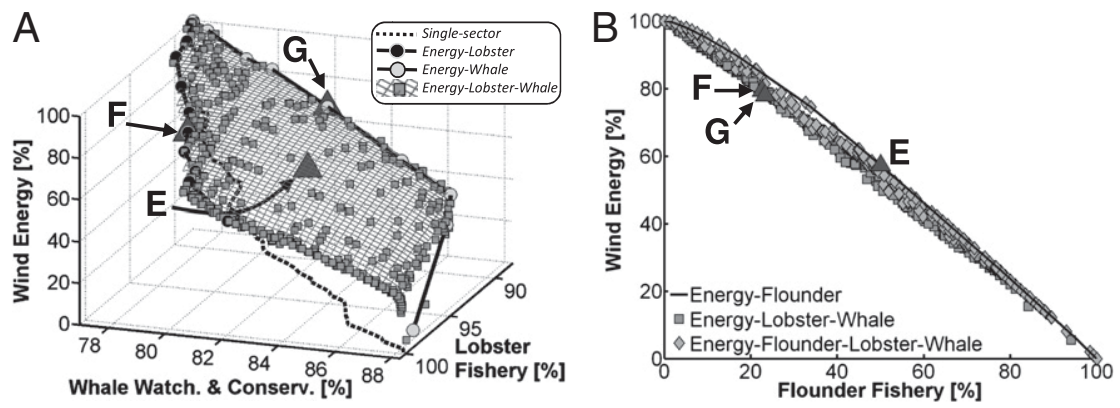

Fig. 3. Tradeoffs in sector values in relation to spatial management strategies. (A) Energy, lobster fishery, and whale-watching sector three-way tradeoff. Solid lines connecting circles (see legend inset) represent pairwise efficiency frontiers shown in Fig. $2 C$ and $D$. Squares and interpolated grid represent the threesector efficiency frontier. Strategy $E$ is beneath the surface (see Fig. 2 for pairwise perspectives). Dashed line indicates outcomes under single-sector management. $(B)$ Energy-flounder fishery tradeoff in relation to pairwise, three-sector, and four-sector efficiency frontiers (see legend inset). Letters and triangles correspond with those in Figs. 2 and 4.

being done in Massachusetts and conservative for what can be expected from MSP planning.

Outcomes under strategic, single-sector management scenarios are illustrated in Fig. $3 A$ by the dashed line, which extends from zero to $100 \%$ energy value and represents energy development in $0,1,2 \ldots 84$ (i.e., all) patches within the energy zones. The line is well beneath the energy-lobster-whale efficiency frontier, indicating that substantial gains to these sectors can be achieved by moving away from single-sector management and toward optimal multisector strategies. However, details of this result are challenging to visualize, even more so in the 4D plot (SI Appendix, Fig. S5). Pairwise tradeoff plots provide more tractable illustrations of the potential value of MSP to each sector. In this case, the dashed line in Fig. $3 A$ is represented in Fig. $2 B-D$ by dashed lines that connect a dark point for each of the 85 single-sector management outcomes.

In relation to the lobster fishery and energy sectors, MSP provides moderate added value over single-sector management (Fig. 2C). Because these sectors value different habitat types, energy development leaves intact many high-value lobster patches. However, single-sector management never reaches the efficiency frontier because the energy sector does not explicitly consider availability of rocky habitat for lobsters, and the presence of a third habitat type (gravel) creates an imperfect tradeoff between hard and soft-bottom habitat in a patch. The true tradeoff between habitats affecting the sectors is accounted for explicitly only under multisector management that seeks to maximize value to both sectors.

MSP provides greater value over single-sector management in relation to whale and energy sectors (Fig. 2D). This is because whale watching is not connected to bottom habitat in the same way that energy or fisheries values are. Single-sector management produces a strong tradeoff because the best wind development sites are unrelated to the spatial distribution of high-value whalewatching areas. This leaves ample options to reallocate wind-energy development and find solutions that are optimal for both sectors.

Because energy and flounder sectors compete for soft-bottom habitat, single-sector management solutions are close to the pairwise efficiency frontier for these two sectors (Fig. $2 B$ ). In relation to just these two sectors, the values of which are strongly linked to a common resource, single-sector management efficiently mediates the tradeoff because loss in habitat to one sector is nearly seamlessly translated into gains to the other. Thus, MSP adds little value over single-sector management. However, value to the flounder fishery from MSP emerges when one also considers the other two sectors, whose resource use patterns are dramatically different. This is illustrated in Fig. $3 B$, where the complete, four-sector efficiency frontier contains points (diamonds) that increase flounder fishery value compared with the three-sector energy-whale-lobster efficiency frontier (Fig. $3 B$, squares). Thus, MSP provides value to the flounder fishery compared with when it is excluded from the multisector analysis. Here, gains are not attributable to improvements over single-sector management but to the ability of MSP to optimally balance wind farm design preferences of the flounder fishery with preferences of other sectors that have different resource requirements. This result emphasizes that MSP should be comprehensive and inclusive (i.e., ecosystem-based), minimizing losses in value to all directly and indirectly interacting sectors in the system. When more sectors are included in the tradeoff analysis, the gains from MSP become greater.

Vertical and horizontal distances between single-sector and optimal management outcomes indicate the potential valueadded by MSP (Figs. $2 A$ and 4 ). Hump-shaped lines in Fig. 4 indicate that the value of MSP to each sector is greatest when the value of the other sector is near the middle of its range, where the number of options and potential for mediating conflicts is greatest. More generally, the value of MSP increases with the greater number of management strategies that are considered. Thus, enlarging the provisional energy zones is expected to enhance the potential value of MSP, and in general, MSP will create the greatest benefits when done at a large scale (e.g., ecosystemscale). Of course jurisdictional, logistical, data and other constraints may set an upper bound on the scale of MSP.

Strategic spatial planning has the potential to increase (or prevent losses in) flounder, lobster, and whale sector values by up to $\sim 1 \%, 4 \%$, and $5 \%$ respectively, at no cost to the energy sector [Fig. $4 A$ and associated table (Fig. 4, Lower)]. Although small, these percentages reflect substantial monetary, cultural, and conservation benefits. Over the expected 27-y planning horizon, the fisheries are estimated to generate a combined net present value of nearly $\$ 3$ million just within the provisional energy zones in the absence of energy development; for the whalewatching tourism industry, this value may exceed $\$ 30$ million (Fig. 4, Lower, table). Thus, even small percentage gains translate into notable monetary sums. Additionally, requirements for whalepopulation conservation and the cultural importance of whales and fisheries in Massachusetts place a premium on sustaining these sectors in the face of new marine user groups, further emphasizing the value of MSP for reducing conflicts and increasing the value of an ecosystem to society.

Percentage value increases to the energy sector from MSP can be dramatic. Strategically placing turbines in planning units void of rocky habitat enables the energy sector to increase its value by $\sim 7 \%$ over single-sector management at no cost to the lobster fishery (right edge of solid line in Fig. 4B). Allowing for small reductions in values to the fishery and whale-watching sectors enables even larger gains to the energy sector. Given a prescribed maximum impact level on one or more sectors, the energy sector can reach much higher values if it explicitly integrates the needs of the other sectors in siting compared with just filling up its highest value patches until the impact limit is reached. For example, using MSP, the energy sector can increase its value $>10 \%$ with $<5 \%$ impact on the value of the lobster fishery (F in Fig. $4 B$ ). Similarly, allowing no more than $5 \%$ reduction in whale-watching and conservation sector (i.e., $\geq 83 \%$ ), MSP can increase energy value $>25 \%$ (strategy $G$ ). Under more stringent whale-conservation regulations, the value of MSP to the energy sector exceeds $45 \%$. Within the energy zones, maximum net present value to the energy sector could exceed $\$ 30$ billion (Fig. 4, Lower, table). Thus, the energy sector could achieve net savings up to nearly $\$ 14$ billion in present-day dollars through strategic MSP of wind farms in relation to the incumbent sectors in Massachusetts Bay. 
A

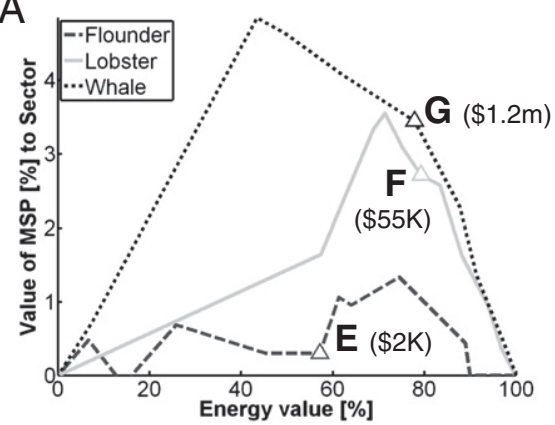

B

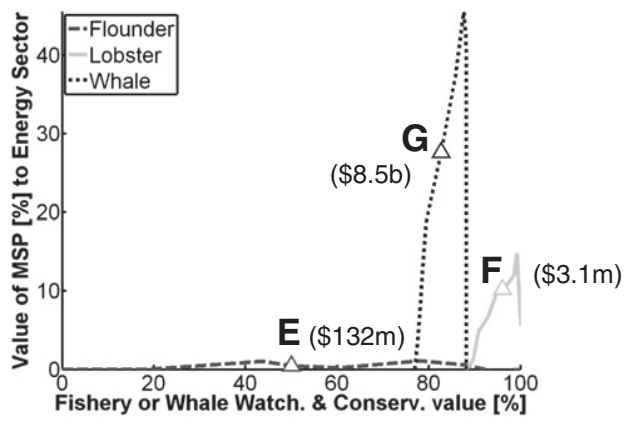

Sector

Flounder

Lobster

Whale

Energy

Maximum absolute sector value

$\$ 682 \mathrm{~K}$

$\$ 2.03 m$

$\$ 33 m$

$\$ 31 b$

Maximum Value of MSP

$1.3 \%, \$ 9 \mathrm{~K}$

$3.6 \%, \$ 72 \mathrm{~K}$

$4.9 \%, \$ 1.6 \mathrm{~m}$

$46 \%, \$ 14 b$

Fig. 4. Value to sectors from MSP over strategic single-sector management, measured for each development scenario as shown in Fig. 2A. (A) Value of MSP to flounder, lobster, and whale-watching sectors in relation to a regulated level of energy development. (B) Value of MSP to the energy sector in relation to regulated target levels of fishery and whale-watching sector values (i.e., $100 \% \mathrm{mi}$ nus a maximum percentage impact allowed). The table shows maximum net present value (NPV) in dollars of each sector alone (for the whale sector, NPV is to the whale-watching tourism industry), within the provisional energy zones (top row); NPVs are multiplied by percentage values of MSP to generate the dollar values in row 2 and for each lettered scenario in the plots. Values in row 2 correspond with the maxima of the curves in $A$ for flounder, lobster, and whale watching and the peak of the dotted line in $B$ for energy.

\section{Discussion}

Concurrent with the decisive steps being taken by governments and industries to promote and develop offshore renewable energy, opposition is growing from coastal residents and marine user groups who fear substantial (some say overestimated) impacts of offshore wind farms on marine ecosystems and services (7). Our MSP approach directly addresses this debate: our case study explicitly quantifies impacts on sectors from energy development and shows how these tradeoffs, and thus people's fears, can be mitigated in Massachusetts Bay. None of the incumbent sectors is immune to negative effects from energy development, and all can benefit from MSP. Across the range of scenarios, the flounder fishery experienced the greatest losses in value (up to $100 \%$ ), yet unnecessary losses were minimized when MSP was used to allocate uses. Other Massachusetts Bay fisheries that use trawls and/or nets over soft-bottom habitat have the potential for similar losses from wind farms and gains from MSP. Percentage losses to the lobster and whale sectors, although smaller than for the flounder fishery, are significant because they translate into substantial absolute changes in monetary value and have critical cultural and conservation implications. Furthermore, MSP greatly improved lobster fishery and whale-watching values over single-sector management, preventing substantial losses. Finally, as one of the most recent user groups to enter marine ecosystems, offshore renewable energy is under tremendous pressure to limit its impact on incumbent sectors (7), while facing obvious internal incentives to maximize its value given high development costs. Our results indicate that MSP can provide substantial guidance toward these twin objectives.

Conflicts over space are becoming the norm in the oceans, and multisector planning is required to reduce these conflicts and optimize marine management (10). Contentious, often subjective, debate over spatial conflicts is expected to rise as ocean uses intensify and expand, further emphasizing the utility of our approach and value of MSP for quantifying and mediating these conflicts.

Resource managers around the world are now in the midst of deciding what MSP will look like, gathering information, developing tools, and attempting to garner buy-in from often skeptical stakeholders (9). Our concrete approach can rationally and objectively identify solutions to the exact kind of problem that resource managers are facing. We offer an efficient, transparent, and transferrable method for comparing management strategies, identifying win-win solutions and avoiding unnecessary conflicts that arise when stakeholders perceive tradeoffs that do not actually exist. By demonstrating how MSP works and quantifying its value over conventional management, these results may enhance stakeholder and decision-maker buy-in to MSP.

The efficiency frontier, although familiar to economists, has seldom been applied to marine resource management (4). However, its flexibility and simplicity make it a promising tool for decision-makers. Several features add to its utility. First, it is not necessary to characterize sector values in a single currency, such as dollars. Instead, the merits of different decisions can be compared based on changes in sectoral values (in absolute or percentage terms), allowing comparison of very different ecosystem services, including those [e.g., recreational opportunities, nutrient cycling (3)], that rely on nonmarket values, such as aesthetics or conservation. Second, plotting potential solutions relative to the efficiency frontier is a powerful method for visualization and communication, allowing decision-makers to compare many alternatives simultaneously. Although a sector-weighting scheme (e.g., an indifference curve) may determine a single solution on the efficiency frontier to be "optimal," nearby solutions on the frontier are equally efficient and may be more feasible to implement. This gives decision-makers flexibility to incorporate other considerations (e.g., feasibility, enforceability), selecting a strategy that balances societal preferences and is practical to implement and manage. Additionally, the efficiency frontier can be an effective tool for engaging stakeholders in joint decision-making, highlighting true tradeoffs and serving as a reference point for negotiation. To aid in this, a multidimensional efficiency frontier (Fig. $3 A$ and SI Appendix, Fig. S5) can be deconstructed into pairwise plots (Figs. 2 and $3 B$ ) for visual clarity. Regardless of who holds final decision-making authority, or whose values take precedence, the efficiency frontier guides decisions toward efficient strategies and away from suboptimal ones with unnecessary conflicts. Conversely, without a formal tradeoff analysis to identify the most efficient strategies, management tends to produce outcomes interior to the frontier (6).

In our model, we sought to capture the main drivers of, and tradeoffs among, offshore energy and key ecosystem services that it impacts in Massachusetts Bay. However, a number of simplifying assumptions about the dynamics of these services and the marine ecosystem may influence our results. For example, conservation values other than whales (e.g., birds) are affected by wind turbines. A wind farm also may affect coastal viewshed and property values (4), and its submarine infrastructure may affect fish more than we assumed. Furthermore, other industrial sectors, such as shipping, already have high value in Massachusetts Bay and may have implications for conservation and MSP. Consideration of tradeoffs among these sectors may alter the solutions presented here; therefore, our spatial results should be considered heuristic rather than prescriptive. Finally, although we focused on net present value for directly measuring sector values, we recognize that indirect benefits also exist. Modeling indirect benefits, such as employment and coastal waterfront economic activity, would further enrich our understanding of the value of MSP.

\section{Conclusion}

We offer a transparent and quantitative approach to assessing and communicating ecosystem dynamics and the interactions among 
varied ecosystem services and the sectors they support. The spatially explicit tradeoff analysis we conducted for Massachusetts Bay demonstrates the viability and value of strategic ecosystembased MSP for informing and rationalizing the often entrenched debates around spatial allocation of marine resources, focusing them on objective conflicts and identifying efficient solutions for improving management outcomes. Such a demonstration of the value-added from MSP over sectoral management has been highlighted as one of the most pressing needs for helping move MSP forward in the United States and elsewhere (11). Inertia is a strong force, and when the costs of non-MSP outcomes are undefined, it is easy for decision-makers to succumb to the notion that MSP planning is too difficult or unnecessary. At the same time, institutional inertia can be quickly overcome when a policy window of opportunity is effectively used (12). The introduction of MSP into US National Ocean Policy represents such a policy window and at a time when spatial conflicts over marine ecosystem services are becoming alarmingly prevalent (10). By showing the utility and feasibility of MSP and quantifying its value over conventional management, we provide timely support and momentum for the transition to comprehensive, ecosystem-based management that is needed to address the challenges we face in an increasingly crowded coastal and marine environment.

\section{Methods}

We constructed a spatially explicit, coupled biological-economic model with eight hundred sixty-eight $2 \times 2 \mathrm{~km}$ patches to estimate the spatial distribution and net present value ("value") of four sectors in Massachusetts Bay in response to wind farm development. To keep the analysis tractable, yet realistic, we focused on two energy zones comprising 84 patches. The zones were designated by Massachusetts because they are good wind sites and have fewer potential conflicts with existing sectors than other possible locations (8). We considered the full range of potential development within the zones (i.e., 0 $100 \%$ of patches), with up to eight wind turbines per patch depending on bottom type. These energy zones would still be regulated, even without MSP and under those regulations, the energy sector is expected to strategically design its wind farm to maximize value to its sector. Accordingly, for each leve of wind farm development, we modeled two forms of spatial planning: $(i)$ single-sector, where energy development focused on the most profitable patches for maximizing the value of its sector (SI Appendix, Fig. S3), and fishery and whale-watching sectors tried to maximize values of their own sectors in relation to the chosen wind farm design; and (ii) multisector, where the energy sector coordinated wind farm design with management of the other sectors to maximize the weighted sum of the values of the sectors, or joint value of the ecosystem. The former represents the expected best outcome without MSP; the latter represents the optimal outcome under ecosystem based MSP. The best-case reference scenario is not guaranteed in practice in that management decisions may not be strategic for maximizing individua sector values. Consequently, this comparison provides a conservative estimate of gains from MSP. If single-sector management was less strategic or wind farm design further constrained by other regulations, one would expect larger gains from MSP than shown here.

We considered all major ecosystem and intra- and intersectoral dynamics relevant to the problem using the following assumptions (for full details are given in SI Appendix). Because of cost constraints and impacts from construction noise (i.e., pile driving), wind farm development is limited to soft-bottom habitat. Turbine pylons effectively remove soft-bottom habitat and create a small amount of hard-bottom habitat. During wind farm construction, fishing is excluded from within safety zones ( $1 / 3-\mathrm{km}$ radius) around each turbine, and,

1. McLeod K, et al. (2005) Scientific Consensus Statement on Marine Ecosystem-Based Man agement (Communication Partnership for Science and the Sea, Washington, DC), p. 21.

2. Douvere F, Ehler CN (2009) New perspectives on sea use management: Initia findings from European experience with marine spatial planning. $J$ Environ Manage 90:77-88.

3. Leslie HM, McLeod KL (2007) Confronting the challenges of implementing marine ecosystem-based management. Front Ecol Environ 5:540-548.

4. Lester SE, et al. (2012) Evaluating tradeoffs among ecosystem services to inform marine spatial planning. Mar Policy, in press.

5. Wu JJ, Skelton-Groth K, Boggess WG, Adams RM (2003) Pacific salmon restoration: Trade-offs between economic efficiency and political acceptance. Contemp Econ Policy 21:78-89.

6. Polasky S, et al. (2008) Where to put things? Spatial land management to sustain biodiversity and economic returns. Biol Conserv 141:1505-1524. thus, direct benefits to fisheries are lost in those areas. After construction, mobile-gear fishing remains excluded from within each safety zone.

We linked these assumptions to the fishery sectors via spatially explicit, age-structured lobster and flounder population dynamic models. Population models were themselves integrated with limited-entry fishery fleet models emulative of commercial fisheries management and spatial fishing dynamics in Massachusetts. In the fleet model, each fishery (flounder, lobster) operated as a noncooperative group of fishermen, regulated in the aggregate by exogenously determined fishery rules defining a minimum fish size limit, spatial restrictions in relation to wind farm design, and a total allowable fishing effort level by the fleet. In turn, the fleet allocated fishing effort spatially to generate uniform payoff per unit effort across fished patches. Patch-specific annual payoff to each fishery was based on profits, calculated based on revenues from yields and market price, and costs in relation to fishing effort and fish stock density. We modeled both local (within-patch) and regional (Massachusetts Bay) dynamic processes to calculate the payoff of each fishery within the energy zones (Fig. 1).

We used patch-specific average annual densities of whale-watching tourism boats to calculate payoff in each patch to the whale-watching and conservation sectors. We assumed offshore areas of high use by whalewatching boats correspond with areas of higher whale density important not only for tourism but also for conservation. For this sector, annual payoff is lost near wind turbines during their construction because of the safety zones and noise disturbance that displace boats and whales, respectively. Fishery-whale interactions potentially further reduce payoff because of effects of the lobster fishery on whale mortality (via entanglement with trap lines) and densities (attributable to competition for herring prey that is used as lobster bait).

For the payoff of the energy sector, we estimated potential annual profit in each patch based on estimates of revenue from turbines, determined by number per patch, energy production per turbine, and market price for energy produced, and estimates of costs of turbine construction and maintenance.

For every wind farm design scenario considered, we estimated patchspecific equilibrium annual payoffs to each sector during the periods of wind farm construction and operation and then summed the annual payoffs of each sector across the 84 patches. We then appended the two periods to create a time series of the annual payoffs of each sector within the energy zones over the construction and operation of the wind farm. We amortized these time series with a $5 \%$ economic discount rate, then summed the discounted payoffs to estimate net present value to each sector over the planning horizon of the wind farm scenario, and calculated the percentage value by scaling the net present value of each sector relative to its maximum.

ACKNOWLEDGMENTS. We thank N. Napoli (SeaPlan) and L. Kaufmann (Boston University) for helping frame the MSP problem and for extensive feedback on our study. K. Lagueux and B. Wikgren (New England Aquarium) provided habitat maps. L. Hatch (Stellwagen Bank National Marine Sanctuary) provided whale-watching vessel traffic data. L. Jacobson and A. Johnson (National Oceanic and Atmospheric Administration) assisted with fisheries and whale data, respectively. C. Clark (Cornell University) helped estimate turbine disturbance effects on whales. L. Incze (University of Southern Maine) advised on larval dispersal assumptions. At the University of California, Santa Barbara, K. Selkoe helped frame the study and A. Rassweiller and C. Costello provided technical model assistance. At the National Center for Ecological Analysis and Synthesis (NCEAS), M. Raneletti gathered species demographic values and $\mathrm{S}$. Walbridge provided assistance with geographic information system analysis. N. Baron (COMPASS), C. English (COMPASS), H. Galindo (COMPASS), E. Goldman (COMPASS), K. McLeod (COMPASS), and two anonymous reviewers all gave very helpful editorial comments. Funding was provided by SeaPlan (B.S.H., C.V.K., and C.W.), the Sustainable Fisheries Group (C.W.), and The David and Lucile Packard Foundation through a grant to NCEAS for ecosystem-based management (B.S.H.).

7. Firestone J, Kempton W (2007) Public opinion about large offshore wind power: Underlying factors. Energy Policy 35:1584-1598.

8. Executive Office of Energy and Environmental Affairs (2009) Draft Massachusetts Ocean Management Plan (Executive Office of Energy and Environmental Affairs, Commonwealth of Massachusetts, Boston) Vol 1, pp 1-140.

9. Ehler C, Douvere F (2009) Marine Spatial Planning: A step-by-step approach toward ecosystem-based management (Intergovernmental Oceanographic Commission and Man and the Biosphere Programme) IOC Manual and Guides No. 53, ICAM Dossier No. 6., Paris: UNESCO.

10. McLeod K, Leslie H (2009) Ecosystem-Based Management for the Oceans (Island, WA), p. 368.

11. Halpern B, et al. (2012) Near-term priorities for the science, policy and practice of Coastal and Marine Spatial Planning (CMSP). Mar Policy 36:198-205.

12. Olsson $P$, Folke $C$, Hughes TP (2008) Navigating the transition to ecosystem-based management of the Great Barrier Reef, Australia. Proc Natl Acad Sci USA 105:9489-9494. 